\title{
Satisfacción del profesional de enfermería acerca de un curso de capacitación. Instituto Nacional de Salud. Asunción, Paraguay 2020.
}

\author{
Silvia Sánchez Flecha1 ${ }^{1}$, Mirna Gallardo \\ ${ }^{1}$ Universidad Nacional de Asunción, Facultad de Enfermería y Obstetricia. San Lorenzo, Paraguay \\ ${ }^{2}$ Asociación Paraguaya de Enfermería. Asunción, Paraguay
}

Cómo referenciar este artículo/ How to reference this article:
Sánchez Flecha S, Gallardo M. Satisfacción del profesional de enfermería acerca de curso de capacitación. Instituto Nacional de Salud. Asunción Paraguay 2020. Mem. Inst. Investig. Cienc. Salud. 2021; 19(3): 83-91

\begin{abstract}
RE S U M E N
La capacitación es una actividad que busca mejorar la actitud, conocimiento y habilidades del personal, especialmente de enfermería porque trabaja con personas; enfocándose en: organización, contenidos, facilitadores, ambiente y equipos, entre otros; de modo que el participante sienta colmadas sus expectativas y salga satisfecho de la misma. Este estudio fue observacional, descriptivo, transversal, cuantitativo. El área de estudio fue el Instituto Nacional de Salud de la ciudad de Asunción. El objetivo general fue determinar la satisfacción del profesional de enfermería acerca del curso de capacitación. La muestra la conformó el grupo de profesionales de enfermería que participó del curso, con un muestreo no probabilístico por conveniencia, se aplicó un cuestionario con las variables: características personales y laborales, percepción y opinión. Se tabularon los datos en la planilla Excel 2016 y se analizaron mediante estadística descriptiva, los resultados fueron expuestos en tablas y gráficos. Se cumplieron todos los aspectos éticos. Los resultados fueron: la mayoría tenía entre 23 a 39 años de edad, de sexo femenino, egresada de instituciones privadas, proveniente del interior del país, se enteró de la convocatoria por las redes sociales y se encontraba trabajando, con un solo empleo, en dependencias del Ministerio de Salud Pública y Bienestar Social. Más de la mitad manifestó una percepción positiva y declaró una opinión positiva hacia el curso. Se concluye que los profesionales de enfermería estaban satisfechos con el curso de capacitación. Se recomienda promover capacitaciones, asistencia y organización de eventos.
\end{abstract}

Palabras clave: Satisfacción, capacitación, profesional de enfermería.

\section{Satisfaction of nursing professional about a training course. National Institute of Health. Asuncion, Paraguay 2020.}

\footnotetext{
A B S T R A C T

Training is an activity that seeks to improve the attitude, knowledge and skills of staff, especially nursing because it works with people; focusing on: organization, content, facilitators, environment and equipment, among others; so that the participant feels his expectations are filled and is satisfied with it. This study was observational, descriptive, cross-sectional, quantitative. The study area was the National Institute of Health of the city of Asunción. The overall objective was to determine the satisfaction of the nursing professional about a training course. The sample was made up of the group of nursing professionals who participated in the course, with a non-probabilistic

Fecha de recepción: abril 2021. Fecha de aceptación: octubre 2021

*Autor correspondiente: Silvia Sánchez Flecha. Universidad Nacional de Asunción, Facultad de Enfermería y Obstetricia. Cruzada de la Amistad y 6 de enero. San Lorenzo, Paraguay

Email: silsan64@hotmail.com
} 
sampling for convenience, a questionnaire was applied with the variables: personal and work characteristics, perception and opinion. The data was tabulated in an Excel 2016 worksheet and analyzed using descriptive statistics, the results were exposed in tables and charts. All ethical aspects were fulfilled. The results were: most were between 23 and 39 years of age, female, graduated from private institutions, from the countryside, learned of the call in social media and were working, with a single job, in units of the Ministry of Public Health and Social Welfare. More than half expressed a positive perception and expressed a positive opinion towards the course. It is concluded that nursing professionals are satisfied with the training course. It is recommended to promote training, attending and organization of events.

Keywords: Satisfaction, training, nursing professional.

\section{INTRODUCCIÓN}

La COVID 19 es una enfermedad infecciosa causada por un virus que puede causar diversas afecciones, tales como: el síndrome respiratorio agudo severo: SARS-CoV, (2003) y el MERS-CoV, (2012). Se la ha identificado por primera vez en Wuhan ((provincia de Hubei) China, en diciembre de 2019. Declarada por la OMS como una pandemia, el 11 de marzo de 2020. No requiere un tratamiento especial. Una de cada 6 personas puede desarrollar una enfermedad grave. Los factores de riesgo incluyen: personas mayores, afecciones previas, como obesidad, hipertensión arterial, problemas cardiacos o diabetes, inmunodepresión, embarazo ${ }^{(1-7)}$

Según la Organización Panamericana de la Salud (OPS), en América, las Enfermedades Crónicas No Transmisibles (ECNT) son causa de tres de cada cuatro defunciones. En Paraguay, la obesidad, la diabetes, las enfermedades cardiovasculares y otras ECNT son los principales problemas de salud, conformando una doble carga de enfermedad, caracterizada por la aparición y aumento de las mismas y una serie de factores de riesgo compartidos ${ }^{(8)}$.

El Área Metropolitana de Asunción concentra actualmente más de una tercera parte de la población del país y condiciona estilos de vida que desencadenan factores de riesgo para las ECNT, ya que son más comunes los empleos sedentarios, el consumo de alimentos procesados y la percepción de que caminar es inseguro debido al tráfico, la delincuencia y la falta de espacios para los peatones ${ }^{(9)}$.

Teniendo en cuenta estos datos, se puede presumir que la frecuencia de internación de personas afectadas por COVID 19 con comorbilidades en las Unidades de cuidados críticos, irá en aumento día a día. Sumado a esto, el incremento de casos positivos en el personal de salud, vulnerando el sistema de salud, obliga a la capacitación de los miles de enfermeros que residen en el Paraguay, entre quienes, muchos no ejercen la profesión. Con este adiestramiento se beneficiarán, no solo los pacientes internados en las UCIs, sino también el propio personal de enfermería, quien tiene la oportunidad de acceder a un puesto laboral y otorgar los cuidados con calidad y calidez, tal como caracteriza a Enfermería $(10,11,12)$

Recordando a la Constitución Nacional en su Capítulo VI de la salud, tenemos: Articulo 68. Del derecho a la salud y Articulo 69. Del sistema nacional de salud, los cuales promueven la protección y la promoción de la salud por parte del Estado mediante acciones sanitarias integradas y políticas que las posibiliten ${ }^{(13)}$.

Por lo que, el Ministerio de Salud Pública y Bienestar Social, el Programa Nacional de Becas "Carlos Antonio López", BECAL Paraguay, el Instituto Nacional de Salud, la Asociación Paraguaya de Enfermería (APE) presentaron un proyecto, a través de la fuente de financiación del Banco Interamericano de Desarrollo (BID) con una inversión de US\$ 1.200 .000 en el marco del plan de respuesta nacional a la emergencia sanitaria generada por la pandemia COVID-19, con la finalidad de fortalecer y, por ende, disminuir el impacto negativo que pueda tener sobre el normal funcionamiento del Sistema Nacional de Salud ${ }^{(14,15,16,17)}$

El objetivo del curso fue capacitar al personal de salud (profesionales médicos y de enfermería), en la adquisición de conocimientos, habilidades y competencias que sirvan de apoyo en el manejo de pacientes con diagnóstico de COVID-19 que evolucionaron hasta la fase crítica de la enfermedad; para ello, se organizaron clases 
prácticas con simulaciones en el uso de equipamientos e insumos propios de cuidados intensivos, previamente una parte teórica en plataforma virtual ${ }^{(15,16)}$.

La capacitación continua permite al profesional de enfermería optimizar la práctica profesional, mejorar el nivel de conocimientos, aumentar el valor del currículo, tanto personal como profesional, fortalecer la autoestima, incrementar la posibilidad de promoción interna, entre otros $(18,19,20)$

Ante estos beneficios, diversos estudios han demostrado que el profesional de enfermería se encuentra satisfecho con la capacitación continua, lo que se refleja en la práctica profesional $(18,20,21,22)$.

Actualmente, las practicas han finalizado y ya se han entregado los certificados correspondientes. Dentro de este contexto, fue interesante conocer la satisfacción del profesional de enfermería acerca del curso impartido, ya que el mismo constituye un hito en la historia de la enfermería paraguaya, por ser el primero a nivel nacional y con la expectativa de un gran impacto en la capacitación de estos profesionales. Las respuestas aclaran algunos aspectos y proporcionan sugerencias para mejoras en el futuro.

\section{MÉTODOS Y TÉCNICAS}

Estudio observacional, descriptivo, transversal, cuantitativo. El área de estudio fue el Instituto Nacional de Salud de la ciudad de Asunción. La población la conformó el grupo de profesionales de enfermería participante del curso de capacitación. Se aplicó un muestreo no probabilístico por conveniencia, tomando una muestra compuesta por los sujetos que enviaron las respuestas por correo. La técnica consistió en una encuesta, mediante un cuestionario elaborado en base a los objetivos y las variables del estudio, el cual se remitió al sujeto de estudio a través del correo personal, previamente revisado por tres colegas docentes especialistas en cuidados críticos y urgencias; se aplicó posteriormente, una prueba piloto con los 40 becarios de la primera semana de práctica (de los mismos, solo respondieron 12) a quienes no se tuvo en cuenta para la investigación. Se expusieron los objetivos; se aclaró que no era obligatorio y que podía retirarse del mismo en el momento que quisiera. Se consideró consentimiento informado al acto de responder el cuestionario. Se estimó satisfacción a más de la mitad de respuestas positivas de percepción, utilizándose la escala de Likert para tal fin, considerándose la opción: completamente de acuerdo; y opinión positiva, expresadas. Se cumplió en todo momento con los aspectos éticos de respeto, beneficencia, justicia, tal como se recomienda para las investigaciones con personas humanas. Los datos obtenidos fueron insertados en la planilla Excel 2016, se analizaron los mismos mediante estadística descriptiva y los resultados fueron expuestos en tablas y gráficos. Se elaboraron recomendaciones según conclusiones derivadas.

\section{RESULTADOS}

La gran mayoría de los profesionales de enfermería pertenece al grupo etario de 20 a 39 años de edad, le sigue el grupo de los que poseen entre 40 y 49 años; mayormente, de sexo femenino, principalmente, egresados de una institución privada; una gran mayoría procede del interior del país, en menor cantidad del Departamento Central y los menos, provienen de Asunción; la mayoría, se enteró de la convocatoria por las redes sociales y más de la mitad trabaja en la profesión. Tales datos se pueden visualizar en la Tabla 1. 
Sánchez Flecha S, Gallardo M Satisfacción del profesional de enfermería acerca de curso de capacitación..

Tabla 1. Distribución de profesionales de enfermería según características personales. Instituto Nacional de Salud. Asunción Paraguay 2021. $n=264$

\begin{tabular}{|c|c|c|c|}
\hline $\begin{array}{l}\text { Características } \\
\text { personales del } \\
\text { profesional de } \\
\text { Enfermería }\end{array}$ & Indicadores & Frecuencia & Porcentaje \\
\hline \multirow{4}{*}{ Grupo etario } & 20 a 29 años & 117 & 44 \\
\hline & 30 a 39 años & 117 & 44 \\
\hline & 40 a 49 años & 23 & 9 \\
\hline & 50 años y mas & 7 & 3 \\
\hline \multirow[t]{2}{*}{ Sexo } & Femenino & 211 & 80 \\
\hline & Masculino & 53 & 20 \\
\hline \multirow{3}{*}{ Institución de egreso } & Pública & 57 & 21 \\
\hline & Privada & 187 & 71 \\
\hline & No responde & 20 & 8 \\
\hline \multirow{4}{*}{ Lugar de procedencia } & Asunción & 19 & 7 \\
\hline & Central & 89 & 34 \\
\hline & Otras & 109 & 41 \\
\hline & No responde & 47 & 18 \\
\hline \multirow{7}{*}{$\begin{array}{l}\text { Cómo se enteró de la } \\
\text { convocatoria }\end{array}$} & $\begin{array}{l}\text { Por la prensa (escrita y } \\
\text { televisiva) }\end{array}$ & 23 & 9 \\
\hline & Por parientes y amigos & 14 & 5 \\
\hline & Por colegas & 56 & 21 \\
\hline & Por redes sociales & 118 & 45 \\
\hline & Por página web & 47 & 18 \\
\hline & Correo & 2 & 1 \\
\hline & No responde & 4 & 1 \\
\hline \multirow[t]{2}{*}{ Trabaja } & $\mathrm{Si}$ & 196 & 74 \\
\hline & No & 68 & 26 \\
\hline \multicolumn{2}{|l|}{ Total } & 264 & 100 \\
\hline
\end{tabular}

Fuente: Datos obtenidos por las autoras.

La mayoría de los profesionales de Enfermería posee un solo trabajo; en menor cantidad, están los profesionales que poseen dos empleos, mayormente trabajan en dependencias del MSPBS. La Tabla 2 muestra lo siguiente:

Tabla 2. Distribución de profesionales de enfermería según características laborales. Instituto Nacional de Salud. Asunción Paraguay 2020. $n=196$

\begin{tabular}{llll}
\hline $\begin{array}{l}\text { Características laborales del } \\
\text { profesional de Enfermería }\end{array}$ & Indicadores & Frecuencia & Porcentaje \\
\hline \multirow{2}{*}{ Cantidad de empleos } & Uno & 139 & 71 \\
& Dos & 45 & 23 \\
& Tres & 6 & 2 \\
& No responde & 6 & 4 \\
& MSPBS & 97 & 49 \\
Lugar de trabajo & IPS & 33 & 17 \\
& Sanatorio privado & 31 & 16 \\
& Hospital de & 4 & 2 \\
& Clínicas & & 5 \\
& Universidad & 10 & 11 \\
\hline
\end{tabular}

Fuente: Datos obtenidos por las autoras. 
Sánchez Flecha S, Gallardo M Satisfacción del profesional de enfermería acerca de curso de capacitación..

Más de la mitad de los profesionales de Enfermería expresa una percepción positiva hacia el curso, manifestando, la mayoría, estar totalmente de acuerdo en que es una buena oportunidad de demostrar el valor de la enfermería y a la vez, fortalecer los conocimientos; seguidamente, declaran estar totalmente de acuerdo en que todos los docentes promueven continuamente las medidas protectoras contra la COVID 19 y muestran amplios conocimientos y experiencia; además, están totalmente de acuerdo en que la capacitación recibida fortalece para proceder correctamente $y$, acceder a la misma, fue fácil y sencillo. Esto puede ser observado en la Tabla 3.

Tabla 3. Distribución porcentual de profesionales de Enfermería según percepción acerca del curso. Instituto Nacional de Salud. Asunción Paraguay 2020. $n=264$

\begin{tabular}{|c|c|c|c|c|c|}
\hline Percepción/Opción & $\begin{array}{l}\text { Totalme } \\
\text { nte de } \\
\text { acuerdo }\end{array}$ & $\begin{array}{c}\text { De } \\
\text { acuerdo }\end{array}$ & $\begin{array}{c}\text { En } \\
\text { desacuerdo }\end{array}$ & $\begin{array}{c}\text { Totalmente } \\
\text { en } \\
\text { desacuerdo }\end{array}$ & $\begin{array}{c}\text { No } \\
\text { responde }\end{array}$ \\
\hline $\begin{array}{l}\text { La convocatoria fue } \\
\text { apropiada y resultó } \\
\text { sencillo acceder a ella } \\
\text { Es una buena } \\
\text { oportunidad de demostrar } \\
\text { el valor de la Enfermería }\end{array}$ & 62 & 35 & 2 & 0 & 1 \\
\hline $\begin{array}{l}\text { Es una buena } \\
\text { oportunidad para que el } \\
\text { profesional de Enfermería } \\
\text { fortalezca sus } \\
\text { conocimientos y siga } \\
\text { otorgando cuidados con } \\
\text { calidad y calidez }\end{array}$ & 85 & 15 & 0 & 0 & 0 \\
\hline $\begin{array}{l}\text { Los organizadores } \\
\text { muestran responsabilidad } \\
\text { para el éxito del curso }\end{array}$ & 61 & 39 & 0 & 0 & 0 \\
\hline $\begin{array}{l}\text { Las clases teóricas fueron } \\
\text { impartidas en forma clara }\end{array}$ & 58 & 39 & 0 & 0 & 3 \\
\hline $\begin{array}{l}\text { Las herramientas } \\
\text { utilizadas para las clases } \\
\text { teóricas fueron } \\
\text { adecuadas }\end{array}$ & 56 & 40 & 1 & 0 & 3 \\
\hline $\begin{array}{l}\text { Los docentes teóricos } \\
\text { muestran amplios } \\
\text { conocimientos }\end{array}$ & 73 & 25 & 0 & 0 & 2 \\
\hline $\begin{array}{l}\text { Los docentes técnicos } \\
\text { muestran amplios } \\
\text { conocimientos y } \\
\text { experiencia }\end{array}$ & 75 & 22 & 1 & 0 & 2 \\
\hline $\begin{array}{l}\text { Los docentes se } \\
\text { presentan con uniforme } \\
\text { limpio y presentable }\end{array}$ & 73 & 23 & 0 & 0 & 4 \\
\hline $\begin{array}{l}\text { Todos los docentes } \\
\text { promueven y aplican } \\
\text { continuamente las } \\
\text { medidas protectoras } \\
\text { contra la COVID } 19\end{array}$ & 77 & 22 & 1 & 0 & 0 \\
\hline $\begin{array}{l}\text { Los equipos utilizados } \\
\text { para la práctica fueron } \\
\text { útiles }\end{array}$ & 51 & 39 & 6 & 1 & 3 \\
\hline $\begin{array}{l}\text { La capacitación recibida } \\
\text { me fortalece para } \\
\text { proceder correctamente }\end{array}$ & 68 & 30 & 1 & 0 & 1 \\
\hline
\end{tabular}

Fuente: Datos obtenidos por las autoras. 
La mayoría de los profesionales de Enfermería demuestra una opinión positiva hacia la organización, los docentes teóricos y técnicos y las clases virtuales, no así hacia los elementos y equipos utilizados. La figura 1 nos muestra estos resultados.

Fuente: datos obtenidos por las autoras.

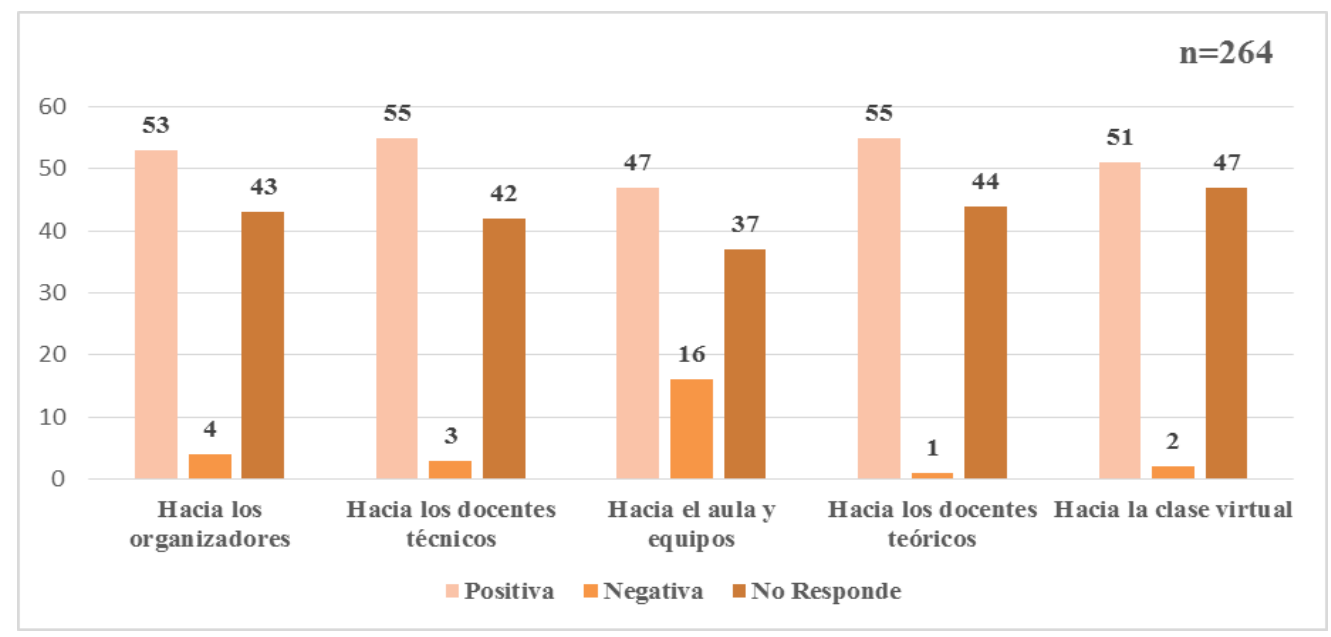

Figura 1. Distribución porcentual de profesionales de enfermería según opinión hacia el curso. Instituto Nacional de Salud. Asunción Paraguay 2020.

Más de la mitad de los profesionales de Enfermería se encuentra satisfecho hacia el curso de capacitación. Esto puede observarse en la figura 2.

Fuente: datos obtenidos por las autoras.

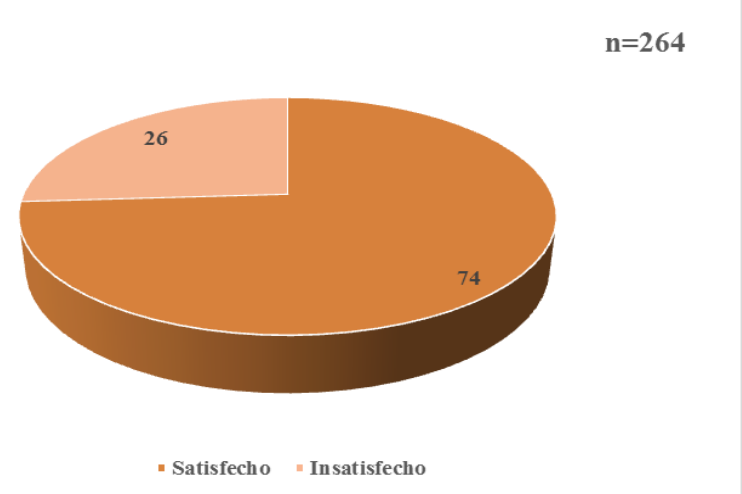

Figura 2. Distribución porcentual de profesionales de enfermería según satisfacción hacia el curso. Instituto Nacional de Salud. Asunción Paraguay 2020.

\section{DISCUSION}

Se examina una muestra de 264 profesionales de enfermería que conforma el $24 \%$ de la población de profesionales enfermeros que asistieron al Curso de capacitación para cuidados críticos a pacientes con COVID 19. Los estadísticos utilizados demuestran una media de 31,6, la mediana de 30, una moda de 29, la desviación estándar de 6,07.

En cuanto a las características personales y laborales, los resultados arrojados por este trabajo, concuerdan con el informe presentado por Silvia Helena De Bortoli Cassiani y colaboradores: La situación de la enfermería en el mundo y la Región de las Américas en tiempos de la pandemia de COVID-19, en donde expone que: a nivel mundial, la fuerza laboral de enfermería es relativamente joven: el $38 \%$ de los profesionales tiene menos de 35 años. También, Vionette Sánchez González en su tesis doctoral: La satisfacción de los estudiantes de enfermería en su primer año de 
estudio de una universidad privada en la integración de la tecnología de información de comunicación (TIC), expone que la mayoría de los participantes es de sexo femenino. Enfermería es una profesión eminentemente femenina y esto se percibe en todos los estudios relacionados a este colectivo (23-25).

En relación a la percepción positiva del profesional de enfermería acerca del curso, no existe evidencia para comparar y discutir estos resultados. La mayoría de trabajos se enfoca en la valoración de la calidad de los cursos, en los factores que intervienen en los profesionales acerca de la asistencia a los mismos, pero no se han encontrado estudios enfocados en la percepción de los enfermeros hacia los cursos de capacitación.

En cuanto a la opinión positiva del profesional de enfermería acerca del curso, coincide con este resultado el trabajo presentado por Julio César Cadena Estrada y colaboradores: Evaluación de la capacitación de enfermería en dos institutos nacionales de salud, quienes concluyeron: La evaluación general del proceso de capacitación es considerada por el personal de enfermería como adecuada, lo cual se refleja en la asistencia anual a cursos. También, Alejandro Hernández Díaz, Mónica Illesca Pretty y Mirtha Cabezas González, de título: Opinión de estudiantes de la carrera de enfermería Universidad Autónoma de Chile, Temuco, sobre las prácticas clínicas, cuya conclusión expresa: Los estudiantes conciben las prácticas clínicas como una metodología activa importante para su formación profesional..., identifican el rol del docente como un elemento preponderante en el escenario clínico. Ante la falta de más estudios previos relacionados a profesionales, se incluye este, teniendo en cuenta que se trata de un colectivo afín $(22,26)$.

Relacionado a la satisfacción del profesional de enfermería hacia el curso; coincide con el estudio de Julio César Cadena Estrada y colaboradores: Evaluación de la capacitación de enfermería en dos institutos nacionales de salud, estudio observacional comparativo y transversal, con una muestra de 415 enfermeras, quienes concluyeron que: la evaluación general del proceso de capacitación es considerada por el personal de enfermería como adecuada, lo cual se refleja en la asistencia anual a cursos. No se encontraron otros trabajos para la discusión (22).

La limitación más importante encontrada en este estudio es la poca colaboración de parte del profesional de enfermería al negarse a participar del mismo, conformando una muestra pequeña que no representa ni siquiera la mitad de la población, motivo por el que los resultados solo pueden reducirse a ella. Quizás en una próxima convocatoria, la situación sea diferente y puedan recabarse datos más amplios. De todas maneras, servirá para continuar esta misma línea de investigación y, además, complementar con otros aspectos, particularmente porque no existe mucha evidencia. Otra limitación significativa es la falta de estudios previos relacionados a la satisfacción del profesional de enfermería sobre la capacitación, pues entorpece la discusión.

\section{AGRADECIMIENTOS}

A la Lic. Nancy Villagra por apoyar y facilitar la ejecución de este trabajo.

A todos los profesionales de Enfermería que se tomaron un momento de su tiempo y respondieron la encuesta.

Conflicto de interés: Las autoras declaran que no existe ningún tipo de conflicto de interés.

Contribución de los autores: Silvia Sánchez Flecha: Planificación y elaboración de la investigación (generación de la idea, elaboración del planteamiento del problema, construcción de los objetivos, escritura del proyecto y recopilación de la bibliografía). Exposición del diseño de investigación y obtención de datos (participación en la toma de datos, tabulación y análisis estadístico de los datos). Elaboración del artículo (argumentación de la introducción del manuscrito, escritura y argumentación con bibliografía de la discusión del manuscrito, redacción del borrador del artículo).

Mirna Gallardo: Análisis y presentación de resultados (exploración y presentación final de los resultados en texto, tablas o figuras). Elaboración del artículo (definición del 
contenido intelectual de la discusión del artículo, redacción de la conclusión a partir de los resultados, revisión del manuscrito).

\section{REFERENCIAS BIBLIOGRAFICAS}

1. Organización Mundial de la salud. Preguntas y respuestas sobre la enfermedad por coronavirus (COVID19). 12 de octubre de 2020. [acceso el 14 de marzo de 2021]. Disponible en: https://www.who.int/es/emergencies/di seases/novel-coronavirus-2019/advicefor-public/q-a-coronaviruses.

2. Organización Mundial de la salud. Los nombres de la enfermedad por coronavirus (COVID-19) y del virus que la causa. 12 febrero de 2021 [acceso el 14 de marzo de 2021]. Disponible en: https://www.who.int/es/emergencies/di seases/novel-coronavirus-

2019/technical-guidance/naming-thecoronavirus-disease-(covid-2019)-andthe-virus-that-causes-it.

3. Organización Panamericana de la Salud. Enfermedad por el Coronavirus (COVID19) febrero de 2021 [acceso el 14 de marzo de 2021]. Disponible en: https://www.paho.org/es/tag/enfermed ad-por-coronavirus-covid-19.

4. Ministerio de Salud Pública y Bienestar Social. Plan de respuesta nacional al eventual ingreso del coronavirus (COVID-19). Marzo 2020 [acceso el 14 de marzo de 2021]. Disponible en: https://www.mspbs.gov.py/dependenci as/portal/adjunto/78806d-

PlandeRespuestaNacionalaleventualingr esodelCoronavirusV1.0.pdf .

5. Ministerio de Salud Pública y Bienestar Social. Cómo evitar el contagio del COVID-19. octubre de 2020 [acceso el 14 de marzo de 2021]. Disponible en: https://www.mspbs.gov.py/portal/2062 8/como-evitar-el-contagio-del-covid19.html .

6. Centros para el control y la prevención de enfermedades. Cómo se propaga el COVID-19. ene del 2021 [acceso el 14 de marzo de 2021]. Disponible en: https://espanol.cdc.gov/coronavirus/20 19-ncov/prevent-getting-sick/howcovid-spreads.html.

7. Ministerio de Salud, Consumo, y Bienestar Social. INFORMACIÓN CIENTÍFICA-TÉCNICA. Enfermedad por coronavirus, COVID-19. ene del 2021 [acceso el 14 de marzo de 2021] Disponible en:

8. https://www.mscbs.gob.es/profesionale s/saludPublica/ccayes/alertasActual/nCo $\mathrm{v}-$

China/documentos/20200404_ITCorona virus.pdf .

9. Gómez C, Guillén C. Enfermedades crónicas no transmisibles (ECNT) y los impuestos al consumo dañino. Fiscalidad para la equidad social jun 2018

[acceso el 14 de marzo de 2021] Disponible en: http://www.decidamos.org.py/fiscalidad Lwp-content/uploads/2018/06/Cap-10Enfermedades-cr\%C3\%B3nicas-notransmisibles-ECNT-y-los-impuestos-alconsumo-da\%C3\%B1ino.pdf.

10. Ministerio de Salud Pública y Bienestar Social. Dirección General de Vigilancia de la Salud. Dirección de Vigilancia de Enfermedades No Transmisibles. Análisis de la Situación de las Enfermedades Crónicas No Transmisibles. Jun 2016 [acceso el 14 de marzo de 2021] Disponible en: http://portal.mspbs.gov.py/dvent/wpcontent/uploads/2016/06/Analisis-dela-Situacion-de-ENT-2015.pdf .

11. ABC Color. Reportan 43 nuevos casos de COVID 19 y suma total llega a 2303. Jul 2020 [acceso el 14 de marzo de 2021]. Disponible en:

https://www.abc.com.py/nacionales/20 20/07/02/reportan-43-nuevos-casosde-covid-19-y-suma-total-Ilega-a-2303/

12. Organización Panamericana de la Salud. Alerta Epidemiológica. Agos 2020 [acceso el 14 de marzo de 2021] Disponible en: file:///C:/Users/usuario/Downloads/202 0-agosto-31-phe-alerta-epi-COVID-19personal-de-salud.pdf .

13. Ministerio de Salud Pública y Bienestar Social. Alerta epidemiológica por aumento de casos de COVID-19 en personal de salud. Sep 2020 [acceso el 14 de marzo de 2021] Disponible en: https://www.mspbs.gov.py/portal/2166 9/alerta-epidemiologica-por-aumentode-casos-de-covid-19-en-personal-desalud.html .

14. Constitución Nacional de la República del Paraguay 1992. junio de 1992. [acceso el 14 de marzo de 2021] Disponible en: https://pdba.georgetown.edu/Parties/P araguay/Leyes/constitucion.pdf .

15. Programa de becas Don Carlos Antonio Lopez. Acuerdo específico de cooperación entre el programa nacional de becas "Don Carlos Antonio López", el Ministerio de Salud Pública y Bienestar Social y la Asociación Paraguaya de Enfermería. Abril 2020 [acceso el 14 de marzo de 2021]. Disponible en: https://www.becal.gov.py/wpcontent/uploads/2020/06/CONVENIO- 
DE-COOPERACION-CON-EL-MSPYBSREF.-AL-COVID-19.pdf .

16. La nación. Becal invierte US\$1.200.000 en capacitación a profesionales de Salud. Abril 2020. [acceso el 14 de marzo de 2021]. Disponible en: https://www.lanacion.com.py/pais/2020 /04/24/becal-invierte-us-1200000-encapacitacion-a-profesionales-de-salud/ .

17. Instituto Nacional de Salud. Capacitación para Médicos y Enfermeras No Intensivistas en Manejo de Pacientes con Covid-19 en el ámbito de Terapia Intensiva. Agos 2020. [acceso el 14 de marzo de 2021]. Disponible en: http://www.ins.gov.py/capacitacionpara-medicos-y-enfermeras-nointensivistas-en-manejo-de-pacientescon-covid-19-en-el-ambito-de-terapiaintensiva/

18. Programa Nacional de Becas de Posgrado en el Exterior "Don Carlos Antonio López". BECAL Médicos y enfermeras inician sus prácticas de capacitación en terapia intensiva el próximo lunes. Jul 2020. [acceso el 14 de marzo de 2021]. Disponible en: https://www.becal.gov.py/medicos-yenfermeras-inician-sus-practicas-decapacitacion-en-terapia-intensiva-elproximo-lunes/.

19. Bejarano AM. Formación y capacitación continua en UCI: ¿Beneficio o sobrecarga laboral? [sede Web]. Buenos Aires. Epidauro. La voz de la Enfermería. Jun del 2018. [acceso el 14 de marzo de 2021]. Disponible en: http://epidauro.com.ar/formacion-ycapacitacion-continua-en-uci-beneficioo-sobrecarga-laboral/ .

20. Metas Estudiantes. ¿Por qué es necesaria la formación continuada? [sede Web]. Valencia España. Enfermeria21 06/05/2018. [acceso el 14 de marzo de 2021]. Disponible en: https://www.enfermeria21.com/conteni do/por-que-es-necesaria-la-formacioncontinuada/

21. Martí Ejarque MM. La formación continuada en enfermería; necesidades y expectativas del personal de enfermería de un hospital universitario de primer nivel. Revista Enfermería Docente [revista en Internet] 2015 julio-diciembre. [acceso el 14 de marzo de 2021]. Disponible en:

http://www.indexf.com/edocente/104pdf/10412.pdf

22. Ponce-Gómez J, Reyes-Morales $H$, Ponce-Gómez G. Satisfacción laboral y calidad de atención de enfermería en una unidad médica de alta especialidad. Rev Enferm IMSS [revista en Internet]
2006. [acceso el 14 de marzo de 2021]. 14(2):65-73. Disponible en: https://www.medigraphic.com/cgi$\mathrm{bin} / \mathrm{new} /$ resumen.cgi? IDARTICULO $=860$ 4

23. Cadena Estrada JC, Olvera Arreola SS, Pérez López MT, Balseiro Almario CL, Matus Miranda R. Evaluación de la capacitación de enfermería en dos institutos nacionales de salud. Enferm. univ [revista en la Internet]. 2012 Sep. [citado 2021 mar 14]; 9(3): 15-24. Disponible

en: http://www.scielo.org.mx/scielo.php?sc ript $=$ sci arttext\&pid=S1665$70632012000300003 \&$ Ing $=$ es

24. De Bortoli Cassiani SH, Munar Jiménez $E F$, Umpiérrez Ferreira $A$, Peduzzi $M$, Leija Hernández $C$. La situación de la enfermería en el mundo y la Región de las Américas en tiempos de la pandemia de COVID-19. Rev Panam de Salud Pública [revista en Internet] 2020 mayo. [citado 2021 mar 14]; Disponible en:

https://scielosp.org/article/rpsp/2020.v 44/e64/es/

25. Sánchez González V. La satisfacción de los estudiantes de enfermería en su primer año de estudio de una universidad privada en la integración de la tecnología de información de comunicación (TIC). Repositorio Institucional de la Universidad de Málaga

26. (RIUMA): riuma.uma.es. [revista en Internet] 2016 diciembre. [citado 2021 mar 14]Disponible en: file:///C:/Users/usuario/Downloads/TD SANCHEZ GONZALEZ Vionette.pdf

27. Zúñiga Careaga Y, Paravic Klijn T. El género en el desarrollo de la enfermería. Rev Cubana Enfermer [Internet]. 2009 Jun [citado 2021 Mar 14]; 25 (1-2). Disponible en: http://scielo.sld.cu/scielo.php?script =sc i arttext\&pid $=$ S086403192009000100009\&lng=es.

28. Hernández Díaz Alejandro, Illesca Pretty Mónica, Cabezas González Mirtha. Opinión de estudiantes de la carrera de enfermería. Universidad Autónoma de Chile, Temuco, sobre las prácticas clínicas. Cienc. enferm. [Internet]. 2013 [citado 202 Mar 14]; 19(1): 131$144 . \quad$ Disponible en: https://scielo.conicyt.cl/scielo.php?scrip $\mathrm{t}=\mathrm{sci}$ arttext\&pid $=\mathrm{S} 0717$ 95532013000100012\&lng=es http://dx.doi.org/10.4067/S071795532013000100012 . 